\title{
Antioxydants naturels végétaux
}

Yohan ROLLAND

Burgundy Botanical Extracts,

Actiparc de Pont de Vaux,

Les Chapelles Sud,

01190 Reyssouze - France

$<$ research@burgundy-extracts.com>

$<w w w$.burgundy-extracts.com>

\begin{abstract}
Oxidation of lipids corresponds to the degradation of fatty acids by a continuation of sequential reactions : the decomposition of peroxides formed on the fatty chains after the attack by radicalaires compounds ends in the production of aldéhydes, cétones, acids, alcohols and go to a loss of the organoleptic and nutritional qualities (characteristic rancy smell and taste), thus a significant loss of economic value. The synthetic antioxidants (BHT, BHA) are effective, but their use is of pleased in more controversial. It is thus advisable to study with attention the plant natural antioxidants. The antioxidizing properties of the plant products are given by a certain number of tests as the test to the DPPH or the test ORAC in aqueous environment, and the measure of the index of peroxides or the Rancimat for the lipophilic phases. Certain tests are above all models of study, and few are really recognized, however certain standards are outlined, and the standardization arrives finally (cf the test ORAC, reference USDA). Among the compound vegetables which stand out very sharply at the level of their antioxidizing property, polyphénols are the most represented in the vegetal reign. It is a very wide family, but the extracts of grape seeds or tea are now well identified, and present results in the fight against the oxidation completely surprising.
\end{abstract}

Key words: oxidation, antioxidant, method of measure, polyphenols, catechins, grape seed, tea, Burgundy

phytothérapie. Ces deux entreprises se sont rapprochées dans le but, pour la première, de valoriser une matière première issue de son activité, les pépins de raisins, et pour la seconde, de s'équiper d'un nouveau potentiel de fabrication d'extraits végétaux à façon. Cette entreprise se développe de manière importante, et compte maintenant 17 salariés, pour un chiffre d'affaires en 2003 de presque 3 millions d'euros.

L'activité de Burgundy est de fabriquer des extraits végétaux, à partir de toute matière première végétale. La capacité d'extraction est d'environ 1500 tonnes de plantes par an, grâce à 4 réacteurs agités à fond filtrant d'une capacité de 24000 litres. Les installations sont compatibles avec l'emploi de tout type de solvants dans un environnement antidéflagrant Eexe II B T 4.

L'usine dispose d'installation de purifications liquide-liquide ou chromatographique, et permet l'obtention en fin de process de composés d'une pureté supérieure à $95 \%$.

Les solvants d'extraction, purifiés ou non, sont concentrés dans un évaporateur-concentrateur sous vide couplé à une colonne de distillation, de $5000 \mathrm{~L}$ en volume utile et $1500 \mathrm{~kg} / \mathrm{h}$ de capacité évaporatoire sur eau, et enfin séché par atomisation dans un atomiseur multiple effet de capacité évaporatoire $80 \mathrm{~kg} / \mathrm{h}$ en eau permettant de produire entre 100 et $150 \mathrm{~kg}$ par heure de poudre fine ou microgranulée. Avant l'industrialisation, les nouveaux produits sont testés sur un équipement pilote, représen-

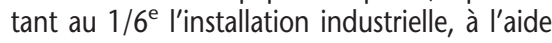
de procédés définis au laboratoire.
L'usine est en cours de certification ISO $9001 / 2000$, avec un plan HACCP répondant à la norme DS 3027 pour l'agroalimentaire.

Maintenant, Burgundy produit des extraits très diversifiés comme par exemple des extraits de germes de soja titrés en isoflavones, extraits d'harpagophytum, guarana, maté, propolis, orthosiphon, kola, etc., pour l'alimentaire, la cosmétique, et de nombreux produits à façon pour la pharmacie.

Les principaux produits, en volume et historiquement, sont les extraits de raisins, pépins ou pépins et pulpes. Ces produits sont très riches en polyphénols et OPC (oligomères procyanidoliques) dont l'activité n'est plus à démontrer. Ce positionnement a permis à Burgundy d'acquérir une grande expérience dans toutes les fractions de cette matière première végétale, et de proposer des produits adaptés à toutes les problématiques: extraction à l'eau ou avec un solvant hydroalcoolique, purifié ou non, mais dans tous les cas, titrés en principes actifs antioxydants.

Autres produits antioxydants très importants chez Burgundy, les extraits de thé vert, plante sélectionnée sur une île d'Indonésie, sont titrés jusqu'à $60 \%$ en dérivés catéchiques sans purification.

Tous ces produits sont exclusivement naturels et présentent une bonne alternative à tous les phénomènes irréversibles d'oxydation. Ils sont dits antioxydants car ils sont plus sensibles à l'oxydation que leur environnement. Faisons un rappel de tous ces éléments pour comprendre les mécanismes d'action mis en jeu. 


\section{Oxydation et anti-oxydants}

L'oxydation est le phénomène qui fait rouiller les métaux, qui fait flétrir les légumes et les fruits, rancir les graisses. II modifie le goût et la couleur des aliments.

L'organisme subit également le phénomène d'oxydation, mais il est équipé pour lutter contre ces altérations: un énorme système de défense est en permanence en place, avec des systèmes enzymatiques et/ou des systèmes de régénération de complexes mettant en jeu par exemple l'acide ascorbique (vitamine $\mathrm{C}$ ) ou le glutathion.

Mais ce système de défense est parfois débordé. Surtout quand les agressions sont multipliées sous l'effet de la fumée du tabac, de la pollution, du soleil, d'un effort physique intense, etc.

Plusieurs cas peuvent engendrer des déséquilibres:

- soit dans des conditions de stress et alors I'oxydation augmente au point de ne pas pouvoir être régulée ;

- soit dans des conditions de mauvaise alimentation et alors les quantités d'antioxydants apportés ne sont pas suffisantes pour rétablir l'équilibre.

C'est là où il y a des dégâts.

\section{L'oxydation est avant tout un phénomène chimique}

L'oxydation est générée par des radicaux libres, espèces chimiques neutres ou chargées instables qui ne cherchent qu'à récupérer un électron dans leur environnement pour retrouver un état plus stable. Ces deux propriétés font que les réactions d'oxydation sont très rapides et se propagent en cascade.

Les espèces moléculaires cibles de l'oxydation sont avant tous les corps gras comme les phospholipides des membranes cellulaires, mais aussi les protéines. Dans le cas des enzymes, l'oxydation entraîne une modification ou perte de l'activité biologique de la molécule, ce qui provoque des désorganisations cellulaires parfois irréversibles entraînant la mort de la cellule. II en est de même quand l'oxydation touche I'ADN ou une partie du système traduction/ transduction.

L'oxygène de l'air à l'état fondamental $\mathrm{O}_{2}$ est peu réactif par rapport à la majeure partie des molécules biologiques. Par contre il existe des formes beaucoup plus réactives et donc plus toxiques (figure 1).

\section{Les mécanismes de l'oxydation}

Traditionnellement, on décrit I'oxydation en trois phases distinctes, mais pratiquement simultanées (figure 2) :

\begin{tabular}{|c|c|c|}
\hline & Radicaux libres: & Espèces à l'origine de radicaux libres: \\
\hline$-\mathrm{O}_{2}^{+}$ & : radical anion superoxyde & $-{ }^{1} \mathrm{O}_{2}$ : oxygène singulet \\
\hline$-\mathrm{OH}^{\bullet}$ & : radical hydroxyle & - $\mathrm{H}_{2} \mathrm{O}_{2}$ : peroxyde d'hydrogène \\
\hline$-\mathrm{HO}_{2}^{*}$ & : radical perhydroxyle & - $\mathrm{ROOH}$ : hydroperoxyde \\
\hline$-\mathrm{RO}^{\circ}$ & : radical alkoxyle (carbonyle excité) & \\
\hline$-\mathrm{ROO}^{\circ}$ & :radical peroxyle & \\
\hline
\end{tabular}

Figure 1. L'oxydation.

1. Initiation : formations d'hydroperoxydes. L'oxygène n'oxyde pas directement les molécules.

Le mécanisme réactionnel initial peut être initié par la chaleur les UV ou les ions métalliques. De même, l'oxygène triplet, stable, peut être activé en oxygène singulet, réactif, par l'intermédiaire d'un photosensibiliateur.

La phase d'initiation aboutit donc à la formation d'espèces très réactives: $\mathrm{ROOH}$ et $\mathrm{R}^{*}$.

2. Propagation : destruction des hydroperoxydes et apparition des composés responsables des goût et odeur de rance.

La liaison 0-O dans un hydroperoxyde est faible et peut être facilement rompue.

Les deux dernières réactions sont en boucles, ce qui explique que des traces d'ions métalliques suffisent à générer une grande quantité de radicaux libres.

Les espèces radicalaires produites par les premières réactions sont hautement réactives et vont à leur tour arracher un hydrogène à une autre molécule ou réagir avec un oxygène triplet.

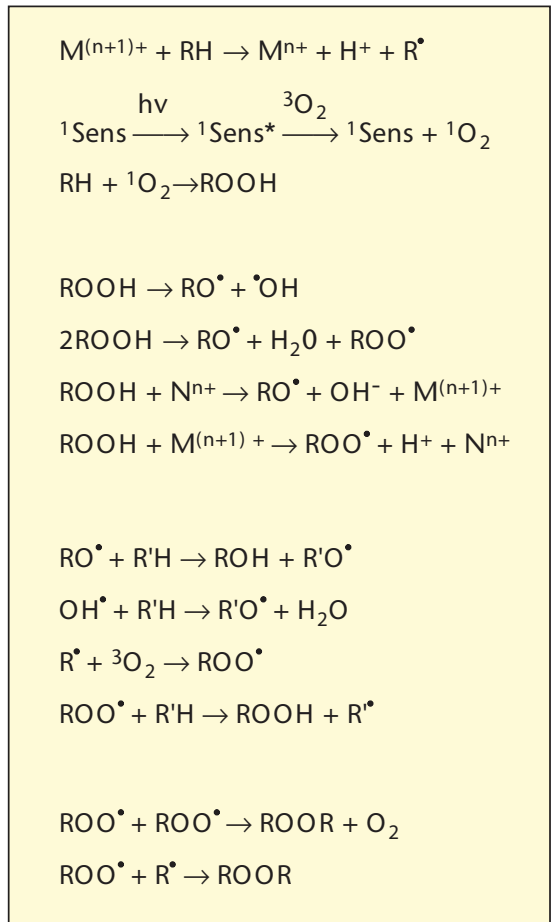

Figure 2. Les phases de l'oxydation.
3. Terminaison arrêt : apparition de nouvelles espèces moléculaires anarchiques.

La chaîne de propagation peut s'arrêter par la formation de polymères ou au contact avec un autre radical. Les molécules créées n'ont plus de fonction biologique.

\section{Les anti-oxydants}

II existe deux types d'antioxydants :

- Les antioxydants primaires ou radicalaires ou vrais, qui permettent l'interruption de la chaîne autocatalytique : $A H+R^{\bullet} \rightarrow A^{\bullet}+R H$. La molécule $\mathrm{AH}$ est antioxydante si le radical formé $A^{\circ}$ est plus stable. La stabilité du radical $A^{\bullet}$ peut $s^{\prime}$ expliquer par sa conversion en composés non radicalaires : $A^{*}+A^{\prime} \rightarrow A-A$ ou $A^{\bullet}+R^{*} \rightarrow A-R$.

- Les antioxydants secondaires ou préventifs qui assurent l'inhibition de la production des radicaux libres. Ce sont des substances décomposant les hydroperoxydes en alcool, des thiols (glutathion, acides aminés soufrés) ou les disulfures, des protecteurs vis-à-vis des UV, comme les carotènes, des chélatants des métaux promoteurs d'oxydation type fer et cuivre, comme l'acide citrique et les lécithines) ou enfin de séquestrants d'oxygène comme l'acide ascorbique.

\section{Les méthodes de mesures de l'oxydation}

Si les mécanismes de l'oxydation sont maintenant bien décryptés et admis par tous, il n'en est pas de même pour les méthodes de mesure de l'oxydation ou du pouvoir antioxydant des principes actifs.

Principale variation, la nature du milieu de l'étude.

En effet, certains composés actifs sont solubles en milieu aqueux (vitamine $C$, polyphénols, etc.), d'autres en milieu lipidique (vitamine $\mathrm{E}$ ). De même, certaines molécules cibles de l'oxydation sont solubles en milieu aqueux (protéines, ADN/ARN) et d'autres en milieux lipidiques (huiles, lipides membranaires).

Les formulateurs de la cosmétique ont bien saisi cet enjeu puisqu'il est d'usage maintenant de protéger les deux phases des émulsions, qu'elles soient eau-dans-huile ou huile-danseau, I'oxydation ayant lieu initialement aux interfaces. 
D'un point de vue nutritionnel, il semble important de jouer sur les deux tableaux, l'organisme étant capable de prendre en charge les composés polaires et apolaires.

Toutes les méthodes de mesure du pouvoir antioxydant d'un principe actif reposent sur le même principe : provoquer une oxydation sur une matrice sensible, et mesurer le ralentissement de dégradation de la matrice protégée par l'antioxydant par rapport à un témoin sans protection.

\section{Mesure en milieu lipidique}

1) Mesure de l'oxydation en temps réel :

La mesure de l'oxydation d'un corps gras en temps réel se fait par la mesure de l'indice de peroxydes. Par définition, l'indice de peroxyde est le nombre de $\mu \mathrm{g}$ d'oxygène actif $\mathrm{du}$ peroxyde contenus dans un gramme de corps gras susceptible d'oxyder l'iodure de potassium avec libération d'iode.

Cette méthode est normalisée (AFNOR, AOCS, UICPA) et représente un critère très utile et très sensible pour apprécier les premières étapes de la détérioration oxydative d'une huile.

Par contre, un résultat faible ne précise pas si le corps gras est de bonne qualité ou s'il est déjà complètement oxydé. II convient de s'assurer de la qualité de la matrice initiale.

L'efficacité de l'antioxydant liposoluble est mesurée par une augmentation plus lente de L'IP par rapport à un témoin sans protection. 2) Mesure de l'oxydation par des tests de vieillissements accélérés :

- Test de Swift ou AOM (Active Oxygen Method) (normalisée AOCS).

Ce test consiste à faire passer un courant d'air purifié dans un échantillon d'huile à $97,8^{\circ} \mathrm{C}$. À des intervalles réguliers, on mesure $\mathrm{I}^{\prime} \mathrm{IP}$, et on détermine le temps nécessaire à un indice de peroxyde de 100 meq d'oxygène actif $/ \mathrm{kg}$ de matière grasse.

Dans le test de Swift modifié (Afnor), le flux de gaz barbote dans une solution de rouge crésol, qui vire au jaune au contact de produits volatils libérés.

- Utilisation de l'appareil «Oxypress». L'échantillon est place dans une étuve hermétiquement close, sous une pression d'oxygène de 3 à 5 bars, à une température de $105^{\circ} \mathrm{C}$. On suit l'évolution de l'oxydation en mesurant la diminution de la pression d'oxygène, correspondant à l'incorporation du gaz dans la matière oxydée (formation d'hydroperoxydes). On enregistre la pression en fonction du temps et on détermine le temps de résistance de l'échantillon préservé par des antioxydant ou non à l'oxydation.

- Utilisation de l'appareil « Rancimat ».

C'est une version automatique du test de Swift, qui permet de déterminer le temps de résistance d'un échantillon à l'oxydation par une mesure conductimétrique.
Un flux d'air fixé à $20 \mathrm{~L} / \mathrm{h}$ traverse un échantillon chauffé à $105^{\circ} \mathrm{C}$. Les composés volatils générés par l'oxydation sont recueillis dans un récipient contenant de l'eau distillée. L'augmentation de la conductivité de l'eau est mesurée et représente la résistance de l'échantillon à l'oxydation (figure 3).

\section{Limite des tests d'oxydation forcée}

Les tests à haute température ne peuvent pas être valablement comparés car les mécanismes mis en jeu sont différents : la vitesse d'oxydation dépend de la concentration en oxygène dont la solubilité croît à haute température et les réactions secondaires de polymérisation, de cyclisation ou de scission deviennent importantes. II n'y a pas de système de vieillissement accéléré idéal, et chaque test doit être adapté au type de matière grasse, au type de milieu. II convient donc de choisir une méthode, $d^{\prime}$ adapter un protocole et de s'y tenir pour la totalité des essais à réaliser.

\section{Mesure en milieu aqueux}

- Le test au DPPH.

LE DPPH (1,1 Diphényl 2 Pycril Hydrazil) est un radical de couleur violet intense.

La mesure de l'efficacité d'un antioxydant (capacité à fixer des radiaux libres, donc arrêter la propagation de la réaction en chaîne) se fait en mesurant la diminution de la coloration violette, due à une recombinaison des radicaux DPPH.

$$
\mathrm{DPPH}_{\mathrm{ox}}+\mathrm{Aox}_{\mathrm{red}} \rightarrow \mathrm{DPPH}_{\text {red }}+\mathrm{Aox}_{\mathrm{ox}}
$$

disparition de la couleur violet à $515 \mathrm{~nm}$ - Le test ORAC

Afin de comparer «l'activité réelle » des nombreux compléments alimentaires apparus aux États-Unis, quelques techniques sont apparues qui évaluent la capacité globale d'antioxydation de mélanges ou d'ingrédients.

La méthode dite «ORAC Value » (Oxygen Radical Absorbance Capacity) mise au point par le Dr CAO de I'USDA (United States Department of Agriculture) en 1993 est très vite devenue la méthode de référence.

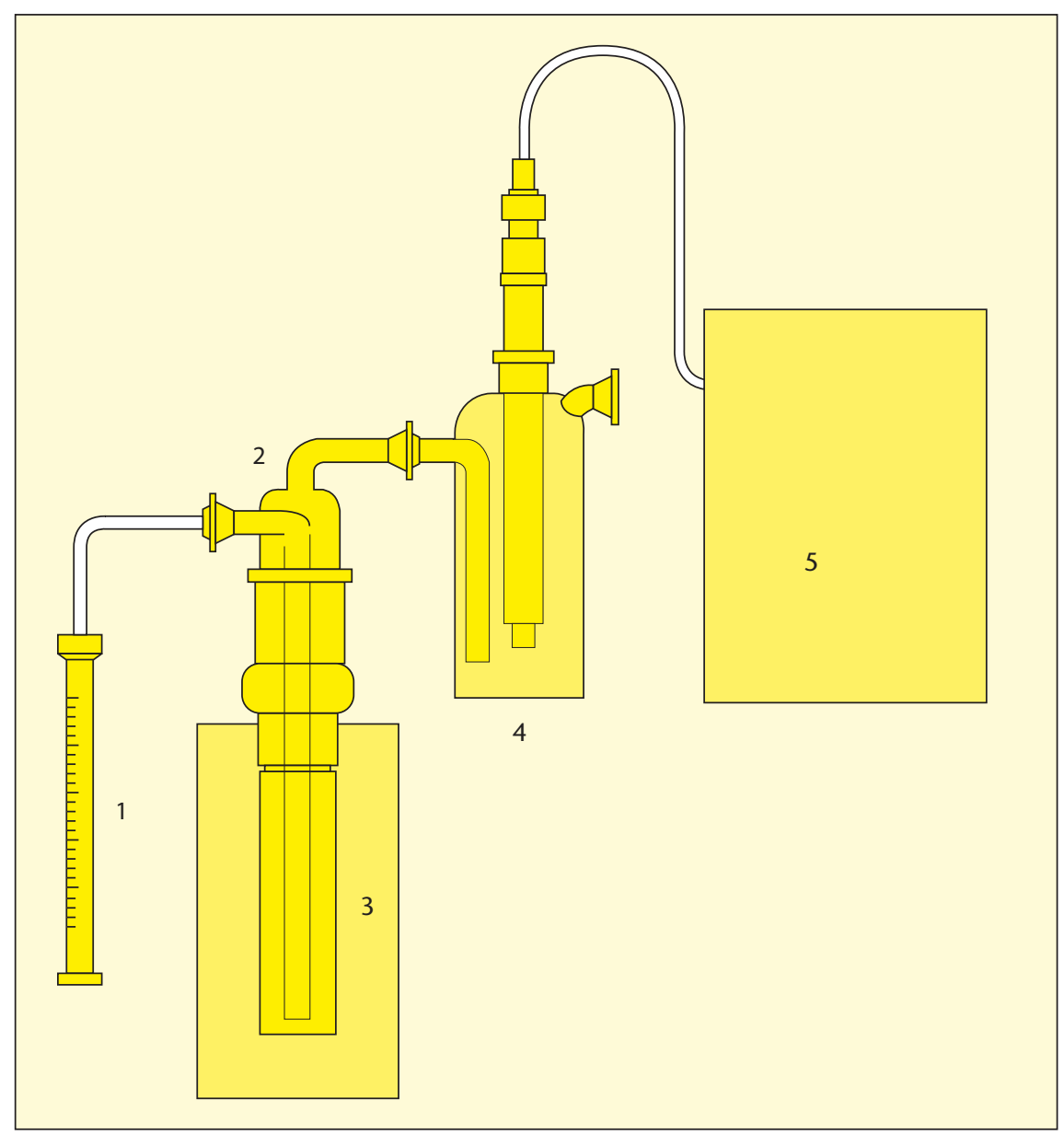

Figure 3. Vue schématique du dispositif d'un test Rancimat. 1) Débitmètre (flux d'air $20 \mathrm{~L} / \mathrm{h}$ ) ; 2) fiole contenant l'échantillon d'huile ou de graisse; 3) bloc de chauffage en aluminium ; 4) récipient contenant l'eau distillée et l'électrode ; 5) amplificateur du signal de conductivité et enregistreur 6 voies. 
Les laboratoires indépendants qui disposent de ce test sont en France Lareal et aux États-Unis Brunswick Lab.

Le principe de cette méthode est la mesure de la destruction d'une protéine végétale particulière qui possède la propriété de fluorescer lorsqu'elle est soumise à un rayonnement lumineux spécifique.

Sous I'action des radicaux libres introduits volontairement dans le milieu réactionnel, la protéine est détruite et perd sa fluorescence.

Lorsqu'un capteur de radicaux libres, tel que un extrait de pépins de raisin, est incorporé dans le milieu, les radicaux libres sont captés, et la fluorescence persiste, donnant ainsi une idée précise du pouvoir anti-radical libre, donc antioxydant, de l'échantillon (figures 4 et 5) :

Relative ORAC value $=$

$$
\begin{gathered}
\frac{\left(A U C_{\text {Sample }}-A U C_{\text {Blank }}\right) \times(\text { molarity of Standard })}{\left(A U C_{\text {Standard }}-A U C_{\text {Blank }}\right) \times(\text { molarity of Sample })} \\
A U C=\text { Area Under Curve }
\end{gathered}
$$

\section{Des éléments pour lutter contre l'oxydation}

L'oxydation est un phénomène irréversible, mais que l'on peut et qu'il faut ralentir. Parmi les solutions, les meilleures sont sûrement issues de la nature, comme les désormais célèbres tocophérols et polyphénols, chacun dans leur domaine de polarité.

Les polyphénols doivent leur activité à, comme leur nom l'indique, un très grand nombre de résidus hydroxyles, qui sont autant de munitions pour lutter contre les radicaux libres et stopper la réaction en chaîne (figures 6 et 7).

Le « French Paradox » est porté en partie par les polyphénols issu du raisin, composés de monomères, de dimères et de molécules encore plus polymérisées (figure 8).

Déjà performant dans les extraits de pépins de raisin à haut pouvoir antioxydant, Burgundy a aussi développé un nouvel antioxydant répondant aux nouvelles exigences réglementaires du secteur des compléments alimentaires qui préconise des solvants d'extraction à l'eau ou à très faible degré alcoolique.

Le Grapemax ExtraPure est extrait sans solvant organique, titre plus de $95 \%$ de polyphénols, et se positionne parmi les extraits les plus efficaces dans le test ORAC.

\section{Résultats des produits}

Les mesures effectuées par un laboratoire indépendant sont présentées au tableau 1.

Les extraits de pépins de raisin sont les plus efficaces dans le test ORAC, parmi les autres

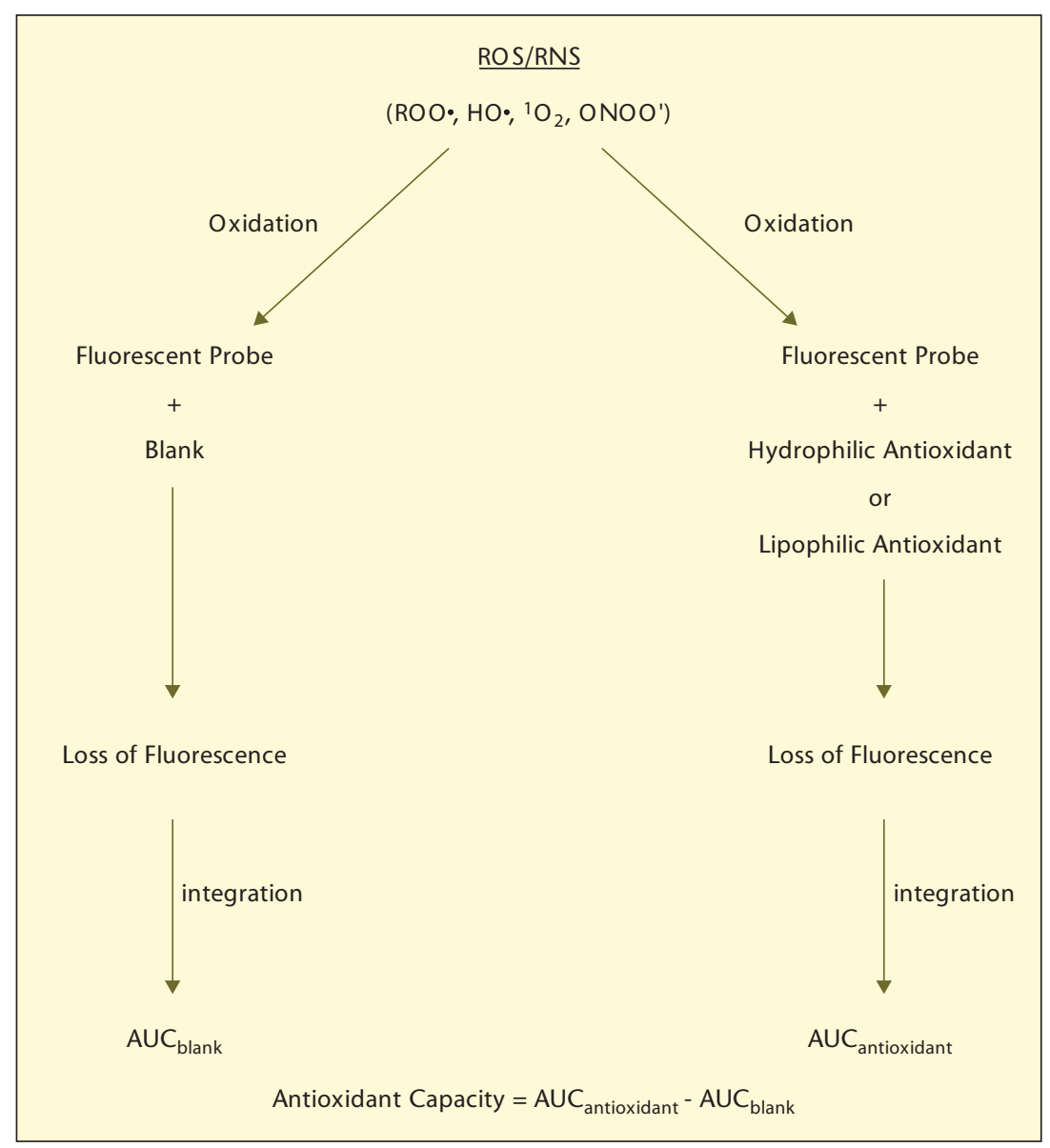

Figure 4. Le test ORAC.

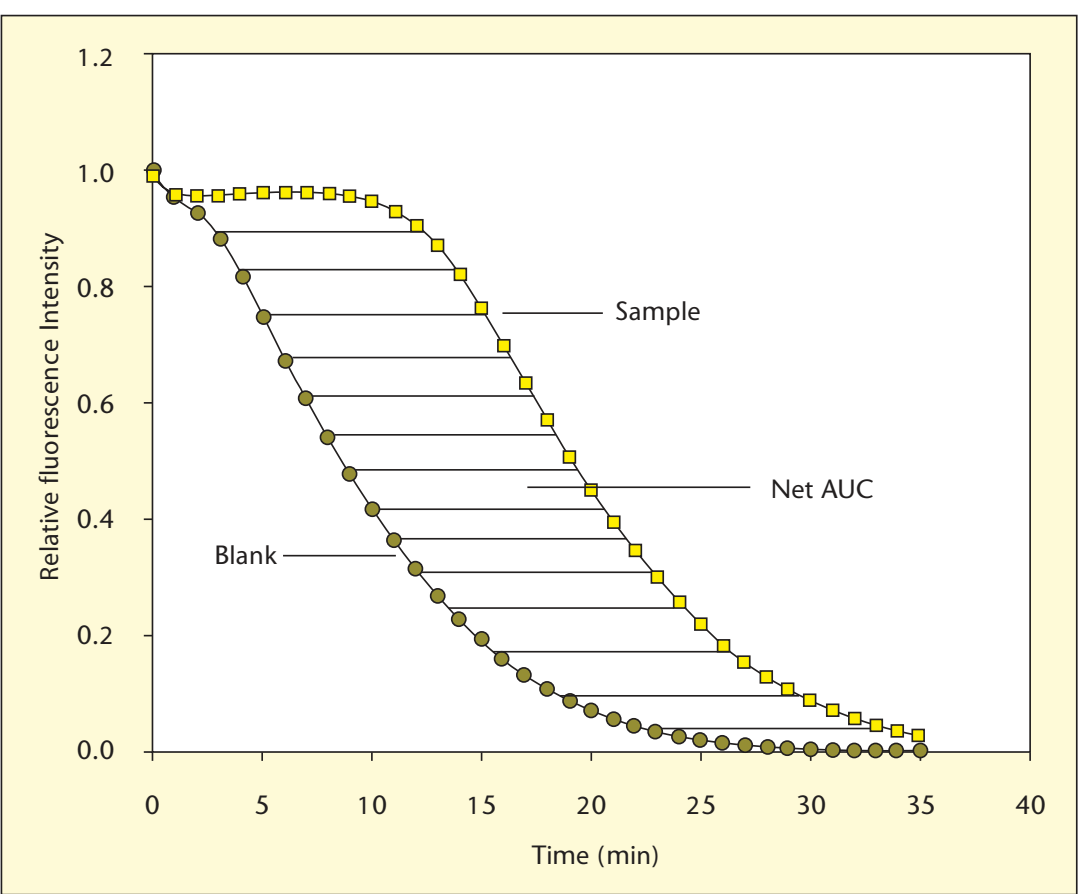

Figure 5. Le test ORAC. 


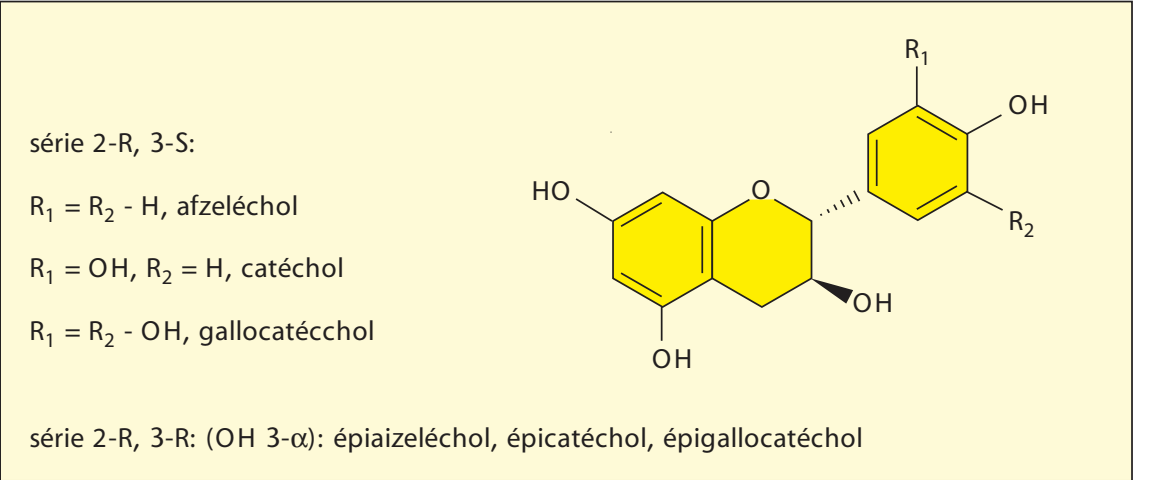

Figure 6.

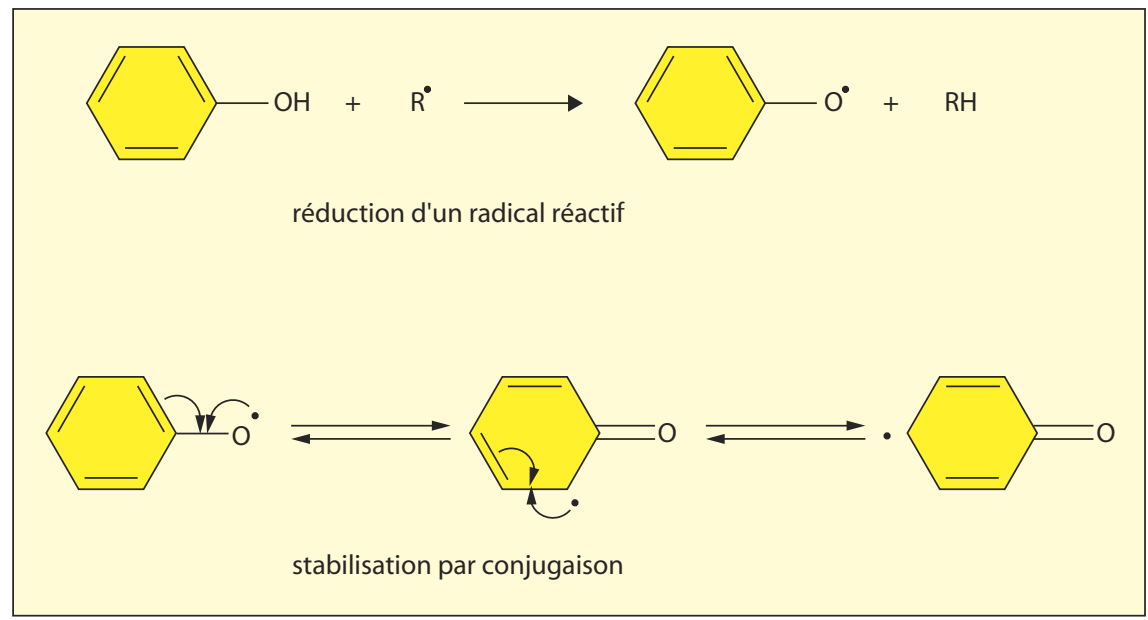

Figure 7. Propriétés réductrices des polyphénols.

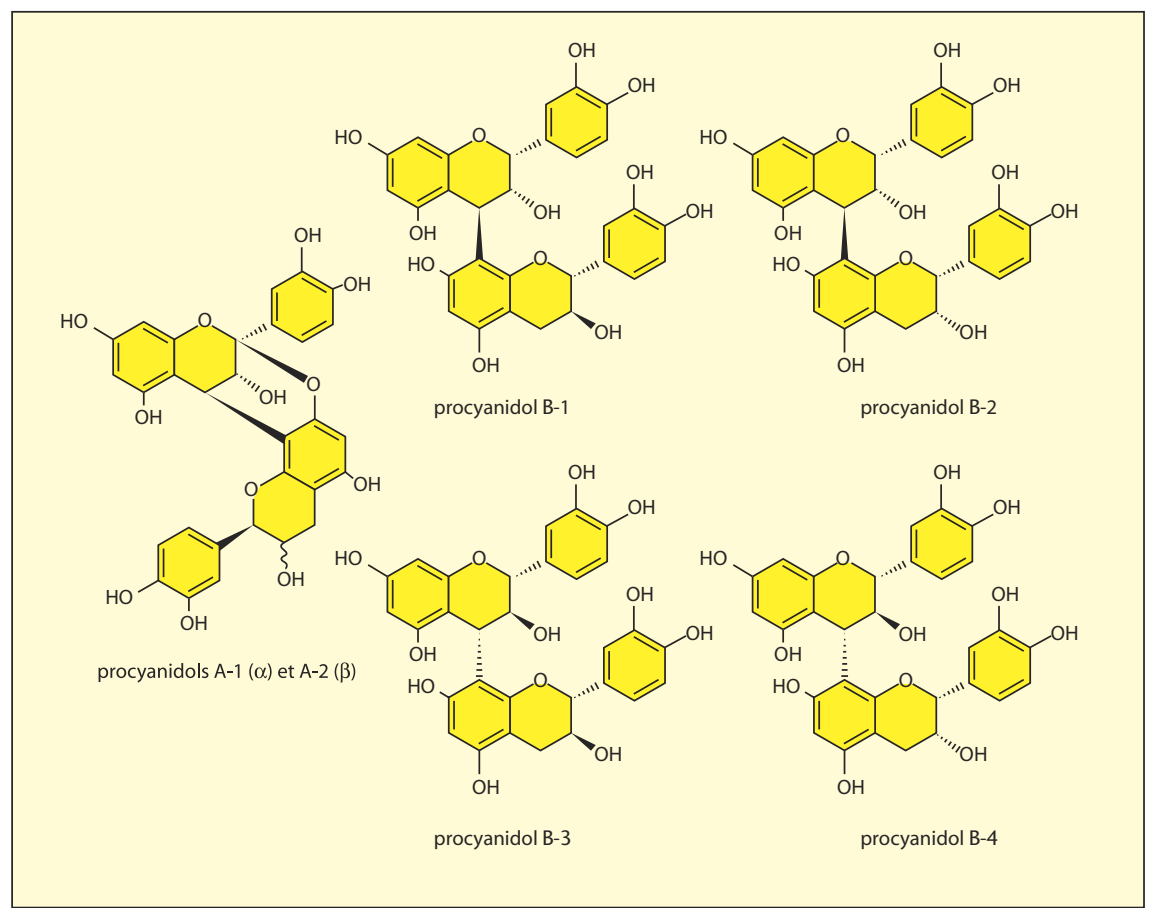

Figure 8. Exemples de structures procyanidoliques dimères.
Tableau 1.

\begin{tabular}{|c|c|c|}
\hline \multicolumn{2}{|l|}{ GRAPEMAX-DE } & $\begin{array}{c}19279 \text { Test ORAC } \\
\text { (Mole TE/g) }\end{array}$ \\
\hline \multicolumn{3}{|c|}{ Extrait purifié de pépins de raisin } \\
\hline \multicolumn{2}{|c|}{ GRAPEMAX-Extrapure } & 18800 \\
\hline \multicolumn{3}{|c|}{ Extrait de pépins de raisin sans solvant organique } \\
\hline \multicolumn{2}{|c|}{ GRAPEMAX-SE } & 8800 \\
\hline \multicolumn{3}{|c|}{ Extrait de pépins de raisin } \\
\hline \multicolumn{3}{|c|}{ GRAPEMAX-BGX } \\
\hline \multicolumn{3}{|l|}{ Extrait de raisin } \\
\hline \multicolumn{3}{|c|}{$\begin{array}{l}\text { Comment situer les Extraits Burgundy par } \\
\text { rapport à : }\end{array}$} \\
\hline Vitamine $\mathrm{E}$ & 1160 & Test ORAC (Mole TE/g) \\
\hline Vitamine C & 5110 & \\
\hline \multicolumn{3}{|c|}{ Autres extraits } \\
\hline \multicolumn{3}{|c|}{$\begin{array}{l}\text { Extrait de thé } 5500 \\
\text { vert }\end{array}$} \\
\hline \multicolumn{3}{|l|}{$\begin{array}{l}\text { Extrait de } \\
\text { Rooibos Tea }\end{array}$} \\
\hline \multicolumn{3}{|l|}{$\begin{array}{l}\text { Extrait de } \\
\text { myrtille } \\
\text { Fruits }\end{array}$} \\
\hline \multicolumn{3}{|l|}{ Fruits } \\
\hline Orange & 24 & \\
\hline Grenade & 106 & \\
\hline Pomme & 14 & \\
\hline \multicolumn{3}{|c|}{ Fruits « transformés " } \\
\hline Jus d'orange & 3000 & \\
\hline Prunes & 40 & \\
\hline Myrtilles & 38 & \\
\hline \multicolumn{3}{|l|}{ Légumes } \\
\hline Ail frais & 2000 & \\
\hline Tomate & 3 & \\
\hline Carotte & 5 & \\
\hline Épinards & 24 & \\
\hline
\end{tabular}

extraits, et bien sûr par rapport aux produits végétaux frais ou transformés.

Plus le produit est purifié en polyphénols (monomères et dimères catéchiques), plus son efficacité antioxydante augmente.

Le Grapemax Extrapure, grâce à des conditions de fabrication spécifique, sans solvant organique, possède une activité antioxydante très élevée qui se place à un niveau équivalent d'un extrait de référence de pépins de raisin purifié.

\section{Conclusion}

L'oxydation est un phénomène complexe, qui met en jeu des espèces moléculaires très réactives et très labiles, ce qui peut rendre son étude difficile.

C'est un phénomène irréversible : il convient de s'assurer de la qualité des matières premières utilisées, de lutter contre l'apparition des facteurs déclenchants (stress, UV, pollution, etc.).

C'est un phénomène qui s'autoalimente : une fois que la réaction est initiée, les réactions en chaîne se poursuivent, et ne s'arrêtent qu'une 
fois toute la matrice oxydée. Seule l'intervention d'agents de terminaison comme des antioxydants peuvent avoir une influence.

Les phénomènes d'oxydation ont lieu dans les milieux aqueux ou lipidiques.

Les mécanismes sont très semblables, et en cas de contact de ces deux milieux (émulsions), l'interface apparaît comme une zone très favorable pour la phase d'initiation.
La nature du milieu à protéger a aussi une influence sur le choix des méthodes de mesures de l'oxydation/du pouvoir anti-oxydant.

Les solutions qui se dégagent pour lutter contre l'oxydation (le retour à un état non oxydé est impossible, mais on peut ralentir la progression) sont de deux ordres :

- agir sur toutes les phases de la matrice,

- utiliser des molécules végétales à haut pouvoir antioxydant.

II ne s'agit plus maintenant de surfer sur la vague de l'impact marketing qui n'est plus novateur, mais d'argumenter sur des vraies efficacités. Les extraits de pépins de raisin restent en Europe et dans le Monde des solutions de choix pour cette application.

Dans la multitude des propositions d'extraits, il faut faire le bon choix de principes actifs dont l'efficacité est réellement mesurée, et parmi tous les tests disponibles, privilégier ceux communément reconnus comme référence. 